\title{
On One Possibility of Closuring the Chain of Equations for Statistical Moments in Turbulence Theory
}

\author{
Edward V. Teodorovich \\ The Institute for Problems in Mechanics, Russian Academy of Sciences, Moscow, Russia \\ Email: teodor@ipmnet.ru
}

Received September 25, 2012; revised October 28, 2012; accepted November 7, 2012

\begin{abstract}
The paper concerns the problem on statistical description of the turbulent velocity pulsations by using the method of characteristic functional. The equations for velocity covariance and Green's function, which describes an average velocity response to external force action, have been obtained. For the nonlinear term in the equation for velocity covariance, it has been obtained an exact representation in the form of two terms, which can be treated as describing a momentum transport due to turbulent viscosity and action of effective random forces (within the framework of traditional phenomenological description, the turbulent viscosity is only accounted for). Using a low perturbation theory approximation for high statistical moments, a scheme of closuring the chain of equations for statistical moments is proposed. As the result, we come to a closed set of equations for velocity covariance and Green's function, the solution to which corresponds to summing up a certain infinite subsequence of total perturbation series.
\end{abstract}

Keywords: Random Velocity Field; Statistical Description; Characteristic Functional; Chain of Equations for Moments; Problem of Closuring

\section{Introduction}

The problem on statistical description of turbulence, even at the simplest assumptions of stationary state and homogeneity of the velocity field, so far remaines to be unsolved in spite of its scientific and practical importance. The point at hand is a search for statistical solution of the Navier-Stokes equation with the availability of external random force (the Langevene force), which simulates the generation of turbulent energy due to the development of instability of large-scale flows at high Reynolds numbers.

Statistical description of turbulent flows means a knowledge of probability to find a given realization of the velocity field $\boldsymbol{u}(\boldsymbol{r}, t)$ in the elementary volume $d[\boldsymbol{u}(\boldsymbol{r}, t)]$ of the space of velocity field realizations that are solutions to hydrodynamic equations (the Navier-Stokes equations) [1,2]. The relevant probabilistic measure $\mu(d[\boldsymbol{u}(\boldsymbol{r}, t)])$ may be written as

$$
\mu(d[\boldsymbol{u}(\boldsymbol{r}, t)])=P[\boldsymbol{u}(\boldsymbol{r}, t)] d[\boldsymbol{u}(\boldsymbol{r}, t)]
$$

where $P[\boldsymbol{u}(\boldsymbol{r}, t)]$ is the functional probability density being subject to the normalization condition

$$
\int P[\boldsymbol{u}(\boldsymbol{r}, t)] d[\boldsymbol{u}(\boldsymbol{r}, t)]=1
$$

where the integration is carried out over all possible velocity field realizations. An experimental determination of the probability density of the velocity field realization is a challenging (in fact impractable) task.

A knowledge of the probability density is equivalent to one of velocity field statistical moments (of all orders). An experimental determination of statistical moments (of finite order) seems to be more actual for approximate describing the field of turbulent velocity pulsations. However, the attemps to obtain equations for statistical moments appear to be unsuccessful since, due to the nonlinearity of Navier-Stokes equations, the equation for statistical moment of given order includes statistical moments of more high orders, and thus an infinite chain of coupled equations arises. It is the chain by Keller and Friedman [3], which is similar to the well-known in statistical physics chain by Bogolyubov-Born-Green-Ivon-Kirkwood [4].

Thus, the problem of cutting off this chain (closure problem) arises. There exist a large body approaches to solving this problem, among which the most simple is neglecting the terms containing moments of order higher then some chosen. Another possibility reduces to attempt to express high-order moments in terms of low-order ones, for example, using the congecture of quasinormality or log-normality, however, this often leads to nonphysical results of a type of negative probability [5].

Another widely used possibility of solving the closure problem reduces to a certain phenomenological hypothesis on connection between high-order and low-order 
moments of the type the hypotheses of turbulent viscosity or of mixing length. As the result, it occurs to be possible to achieve a satisfactory agreement with experimental data in a limited domain of wavenumbers and frequencies.

The method of "direct interaction approximation" proposed by R. Kraichnan [6] (see also [7]) can be treated as a further development of the moment method. Within the framework of this method, in addition to statististical moments, Kraichnan introduces into consideration a function of averaged response of velocity field to external force action (Green's function), which is defined as a solution of the problem on diffusion transfer in a random velocity field at given statistics of the velocity field.

It should be also mentioned the method of constructing a statistical solution to the Navier-Stokes equations using the perturbation theory when the nonlinear term in Navier-Stokes equation is considered as perturbation, and, while culculating statistical moments, a solution for velocity is presented in the form of series in the parameter of nonlinear interaction with subsequent multiplication of series obtained and term-by-term averaging the resulting expression ("simple perturbation theory"). However, in fact the nonlinear term is not small, the dimensionless parameter of nonlinear interaction is defined by Reynolds number, which is very large in the case of fully developed turbulence. Due to this fact, the convergence of perturbation series is slow, i.e. a knowledge of several first terms in perturbation series appears to be not sufficient for estimating the behaviour of turbulent characteristics being a subject of interest. The problem turns out to be similar to that of strong interactions in quantum field theory, where the methods of improving perturbation theory with the help of partial summing up an infinite subsequence of total perturbation series by solving Dysons' equations were developed ("improved perturbation theory"). As applied to description of turbulence, a relevant approach has been proposed by $\mathrm{H}$. Wyld [8]. In his analysis of pertubation series, he used Feynmann's diagram techniques from quantum field theory, where to every term of perturbation series there are assigned some graphic symbols with prescribed pictorial physical meaning as processes of quanta production, annihilation or propagation. Note that the effective Reynolds number defined as a ratio of energy input to given mode from large-scale modes to the value of viscous absorbtion caused by interaction with smale-scale modes appears to be approximately unit under the conditions of stationary developed turbulence. This provides more rapid convergence of perturbation series. Also note that, within the framework of "improved perturbation theory", the pertubation method is used for finding some quantities connected with a solution (for example, the self-energy operator in quantum field theory). A substitution of this quantity into the equation and sequent solving that corresponds to summing up a certain infinite subsequence of total perturbation series.

It should be also pointed out an alternative possibility to improve the perturbation theory related to application of the renormalization-group (RG) method (see reviews $[9,10])$, this approach corresponds to summing up a certain (but yet another) infinite subsequence of total series.

One more peculiarity of developed turbulence, which enables one to reveal the key features of the energy spectrum of turbulent velocity pulsations in some wavenumber range, is a conjecture that the turbulent spectrum is formatted due to nonlinear interactions between modes of close scales, whereas interactions between modes with significantly different scales are performed through the cascade sequence of interactions between modes of all intermediate scales (Richardson's cascade), in other words, one has to tell about "a locality of intermode interactions in the wave-number space" [11]. This is explained by the fact that the interaction between modes of significantly different scales reduces to a simple mechanical translation of small-scale modes by large-scale ones without energy redistribution [12]. This brings up the problem of separating strong interactions between modes with essentially different scales, which reduce to translation, from weak interactions between modes of close scales that execute the energy transfer along the wave-number spectrum [13].

The concept of locality of intermode couplings lies at the basis of describing turbulence within the framework of renormalization-group method [14], and, in particular, the method of $\varepsilon$-expansion, which makes an integral part of the RG-technique, should be treated as a way of separating off local interactions from nonlocal interations, which do not take part in formatting the spectrum [14].

The hypothesis of locality enables one to predict the shape of energy spectrum in a certain wave-number range (Kolmogorov's spectrum), which turns out to be in good agreement with experimental data.

The present investigation aims to obtain a closed set of equations for two functions of direct interest, namely, the covariance of velocity field and the function of averaged responce to external force action (Green's function). Similarly to the formulation of turbulence theory in terms of statistical moments, in the approach proposed, a chain of equations for quantities through which statisticl moments of velocity field can be expressed arises. A closure of this chain is performed by using the perturbation theory at a certain stage. This enables one to obtain a set of equations for two scalar functions. A solution to these equations corresponds a sum of some infinite subsequence of the total pertubation-theory series. The method of space-time characteristical functional and the equation in functional derivatives for that are used in probabilistic 
description of turbulent velocity field.

\section{Mathematical Statement of the Problem}

One way of describing random processes and fields is the method of characteristic functional. In turbulence theory the space-time characteristic functional, which has been first introduced R.Lewis and R.Kraichnan [15], is a functional Fourier-transform of the probability density of a random velocity field [1, Ch.3]

$$
\begin{aligned}
& W\left[\eta_{\alpha}(\boldsymbol{r}, t)\right]=\left\langle\exp \left\{i \int \eta_{\alpha}(\boldsymbol{r}, t) u_{\alpha}(\boldsymbol{r}, t) \mathrm{d} \boldsymbol{r} \mathrm{d} t\right\}\right\rangle \\
& \equiv \int P[\boldsymbol{u}(\boldsymbol{r}, t)] \exp \left\{i \int \eta_{\alpha}(\boldsymbol{r}, t) u_{\alpha}(\boldsymbol{r}, t) \mathrm{d} \boldsymbol{r} \mathrm{d} t\right\} \mathrm{d}[\boldsymbol{u}(\boldsymbol{r}, t)]
\end{aligned}
$$

here $\eta_{\alpha}(\boldsymbol{r}, t)$ are the components of arbutrary vector function of space and time coordinates, $P[\boldsymbol{u}(\boldsymbol{r}, t)]$ is the functional probability density of realizing velocity field $\boldsymbol{u}(\boldsymbol{r}, t)$ and integration is carried out over all velocity field realizations allowed by the set of equations for this field.

To made the record more simple, we will further use digital notations for a set of coordinates of space-time point and subscripts of vector or tensor component $\left\{\boldsymbol{r}_{1}, t_{1}, \alpha_{1}\right\} \equiv 1$, according to which $u_{\alpha_{1}}\left(\boldsymbol{r}_{1}, t_{1}\right)=u(1)$, and integration over space-time coordinates and summing over component numbers with repeated digital notations will be implied (the Eistein rule), i.e.

$$
u(1) v(1)=\sum_{\alpha_{1}} \int \mathrm{d} r_{1} \mathrm{~d} t_{1} u_{\alpha_{1}}\left(\boldsymbol{r}_{1} t_{1}\right) v_{\alpha_{1}}\left(\boldsymbol{r}_{1} t_{1}\right)
$$

When the vector or tensor subscripts are written explicitly, the digital notation will only relate to space-time coordi- nates.

According to these notation

$$
W[\eta(1)]=\langle\exp \{i \eta(1) \cdot u(1)\}\rangle
$$

From the definition of characteristic functional it follows that statistic moments of velocity field are expressed as functional derivatives of the functional $W$ with respect $\eta(1)$ at $\eta=0$.

$$
\begin{aligned}
& \langle u(1)\rangle=\left.\frac{\delta}{i \delta \eta(1)} W[\eta]\right|_{\eta=0} \\
& \langle u(1) u(2)\rangle=\left.\frac{\delta^{2}}{i \delta \eta(1) i \delta \eta(2)} W[\eta]\right|_{\eta=0}
\end{aligned}
$$

and so on. Semi-invariants (cumulants or irreducible means) of the velocity field are defined through functional derivating the characteristc functional logarithm $\ln W$.

After excluding the pressure by means of the equation of continuity, the Navier-Stokes equation can be written in the form

$$
\begin{aligned}
& L^{(0)}(1,2) u(2) \\
& +\frac{1}{2} V(1 \mid 2,3) u(2) u(3)=f(1)+F(1)
\end{aligned}
$$

here

$$
L^{(0)}(1,2)=\left[\partial_{t}^{(1)}+v_{0} \Delta^{(1)}\right] \delta(1-2)
$$

$f(1)$ is the component of external regular force, $F(1)-$ the component of external random force specified statistically, $\Delta^{(1)}$ is the Laplace-operator construted of derivatives with respect to components of the vector $\boldsymbol{r}_{1}$,

$$
\begin{aligned}
V(1 \mid 2,3)= & V_{\alpha_{1} \alpha_{2} \alpha_{3}}\left(\boldsymbol{r}_{1}, t_{1} \mid \boldsymbol{r}_{2}, t_{2} ; \boldsymbol{r}_{3}, t_{3}\right) \\
= & -\left[P_{\alpha_{1} \alpha_{2}}(1,4) \partial_{\alpha_{3}}^{(2)}+P_{\alpha_{1} \alpha_{3}}(1,4) \partial_{\alpha_{2}}^{(3)}\right] \\
& \cdot \delta(4-2) \delta(4-3)
\end{aligned}
$$

here

$$
P_{\alpha_{1} \alpha_{4}}(1,4)=\delta_{\alpha_{1} \alpha_{4}} \delta\left(\boldsymbol{r}_{1}-\boldsymbol{r}_{4}\right)-\partial_{\alpha_{1}}^{(1)} \partial_{\alpha_{4}}^{(1)} \Delta^{-1}\left(\boldsymbol{r}_{1}-\boldsymbol{r}_{4}\right)
$$

is the operator of transversal projection satisfied the condition

$$
\partial_{\alpha_{1}}^{(1)} P_{\alpha_{1} \alpha_{4}}\left(\boldsymbol{r}_{1}-\boldsymbol{r}_{4}\right)=0
$$

$\Delta^{-1}\left(\boldsymbol{r}_{1}-\boldsymbol{r}_{4}\right)$ is the reverse Laplace operator defined by the relation

$$
\Delta^{(1)} \Delta^{-1}\left(\boldsymbol{r}_{1}-\boldsymbol{r}_{4}\right)=\delta\left(\boldsymbol{r}_{1}-\boldsymbol{r}_{4}\right)
$$

i.e. Green's function for Laplace operator.

If there exists external regular force $f$, the characteristic functional will be a functional of two functional arguments $\eta$ and $f$.

While calculating the characteristic functional the averaging is carried out over external random force $F$. Without loss of generality one may assume that this force is solenoidal and statistically described by centered normal distribution with a covariance of the form

$$
D_{\alpha_{1} \alpha_{2}}^{(0)}(1,2)=P_{\alpha_{1} \alpha_{2}}(1,3) D^{(0)}(3-2)
$$

Under these assumptions and using a representation of characteristic functional in the form of two-fold functional integral one can obtain for that an equation in functional derivatives $[16,17]$

$$
\begin{aligned}
& L^{(0)}(1,2) \frac{\delta W[\eta, f]}{i \delta \eta(2)} \\
& +\frac{1}{2} V(1 \mid 2,3) \frac{\delta}{i \delta \eta(3)} \frac{\delta W[\eta, f]}{i \delta \eta(2)} \\
& -i D^{(0)}(1,2) \frac{\delta W[\eta, f]}{\delta f(2)}=f(1) W[\eta, f] .
\end{aligned}
$$

This equation is an analogous to Hopf's equation for spatial characteristic functional [1]. 
In addition we present the equation for $\ln W$

$$
\begin{aligned}
& L^{(0)}(1,2) \frac{\delta \ln W}{i \delta \eta(2)}+\frac{1}{2} V(1 \mid 2,3) \frac{\delta}{i \delta \eta(2)} \frac{\delta \ln W}{i \delta \eta(3)} \\
& -i D^{(0)}(1,2) \frac{\delta \ln W}{\delta f(2)} \\
& +\frac{1}{2} V(1 \mid 2,3) \frac{\delta \ln W}{i \delta \eta(2)} \frac{\delta \ln W}{i \delta \eta(3)}=f(1)
\end{aligned}
$$

Now let us turn from variables $\eta$ and $f$ to new functional variables $\hat{\eta}$ and $\hat{f}$ definded by relations

$$
\begin{aligned}
& \hat{\eta}(1)=\delta \ln W[\eta, f] / i \delta \eta(1), \\
& \hat{f}(1)=\delta \ln W[\eta, f] / i \delta f(1)
\end{aligned}
$$

When $\eta \rightarrow 0$, we get $\hat{\eta}(1)$ tends to $\langle u(1)\rangle$, which is a mean value of velocity component $\alpha_{1}$ at the space- time point $\left\{\boldsymbol{r}_{1}, t_{1}\right\}$ in the external force field $f_{\alpha_{2}}\left(\boldsymbol{r}_{2}, t_{2}\right)$.

Going to new variables can be performed with the help of functional Legendre transformation through introducing new characteristic functional

$$
\Psi[\hat{\eta}, \hat{f}]=-\ln W[\eta, f]+i \eta \hat{\eta}+i \hat{f f}
$$

In this case

$$
\frac{\delta \Psi}{i \delta \hat{\eta}(1)}=\eta(1), \quad \frac{\delta \Psi}{i \delta \hat{f}(1)}=f(1)
$$

The limit $\eta \rightarrow 0$ corresponds to the exremality condition of the functional $\Psi: \delta \Psi / i \delta \hat{\theta}=0$, whereas the extremality condition of functional $\Psi$ with respect to $\hat{f}$ corresponds to absense of external regular force.

The functional $\Psi[\hat{\eta}, \hat{f}]$ obeys the following equation in functional derivatives

$$
\begin{aligned}
& L^{(0)}(1,2) \hat{\eta}(2) \\
& +\frac{1}{2} V(1 \mid 2,3)\left[\hat{\eta}(2) \hat{\eta}(3)+\frac{\delta \hat{\eta}(3)}{i \delta \eta(2)}\right] \\
& -i D^{(0)}(1,2) \hat{f}(2)=\frac{\delta \Psi}{i \delta \hat{f}(1)}
\end{aligned}
$$

In the limit $\eta \rightarrow 0$, Equation (2.17) transforms into the equation for mean velocity field with accounting for Reynolds tension.

For further analysis the mixed derivatives should be considered, namely,

$$
\begin{aligned}
& \frac{\delta^{2} \Psi}{\delta \eta(2) i \delta \hat{\eta}(1)}=\frac{\delta \hat{\eta}(3)}{\delta \eta(2)} \frac{\delta^{2} \Psi}{\delta \hat{\eta}(3) i \delta \hat{\eta}(1)} \\
& +\frac{\delta \hat{f}(3)}{\delta \eta(2)} \frac{\delta^{2} \Psi}{\delta \hat{f}(3) i \delta \hat{\eta}(1)}=\delta(1-2)
\end{aligned}
$$

$$
\begin{aligned}
& \frac{\delta^{2} \Psi}{\delta \eta(2) \delta \hat{f}(1)}=\frac{\delta \hat{\eta}(3)}{\delta \eta(2)} \frac{\delta^{2} \Psi}{\delta \hat{\eta}(3) \delta \hat{f}(1)} \\
& +\frac{\delta \hat{f}(3)}{\delta \eta(2)} \frac{\delta^{2} \Psi}{\delta \hat{f}(3) \delta \hat{f}(1)}=0
\end{aligned}
$$

Here

$$
\delta(1-2) \equiv \delta_{\alpha_{1} \alpha_{2}} \delta\left(\boldsymbol{r}_{1}-\boldsymbol{r}_{2}\right) \delta\left(t_{1}-t_{2}\right)
$$

If the extremality conditions for functional $\Psi$ are satisfied, the expression that includes functional derivatives of functional $\Psi$ only with respect to fields $\hat{\eta}$ vanish as well as functional derivatives of functional $\ln W$ only with respect to fields $f$. This exact property can be easily proved within the framework of diagramm technique [14].

In particular

$$
\frac{\delta^{2} \Psi}{\delta \hat{\eta}(1) \delta \hat{\eta}(2)}=0, \quad \frac{\delta^{2} \ln W}{\delta f(1) \delta f(2)}=0
$$

In this case one will have

$$
\begin{aligned}
& \frac{\delta \hat{\eta}(1)}{i \delta \eta(2)}=\frac{\delta}{i \delta \eta(1)} \cdot \frac{\delta}{i \delta \eta(2)} \ln W \rightarrow C(1,2), \\
& \frac{\delta \hat{f}(1)}{\delta \eta(2)} \ln W=\frac{\delta}{\delta f(1)} \frac{\delta}{i \delta \eta(2)} \ln W \rightarrow G(2,1)
\end{aligned}
$$

Here $C(1,2)=\langle u(1) u(2)\rangle-\langle u(1)\rangle\langle u(2)\rangle$ is the covariance function of velocity field and $G(2,1)$ is Green's function that describes averaged response of velocity field at the space-time point 2 to unit force action localizated at point 1 .

From Equations (2.18) and (2.19) we obtain

$$
\begin{aligned}
& \frac{\delta^{2} \Psi}{\delta \hat{f}(1) \delta \hat{\eta}(2)}=i G^{-1}(1,2) \\
& \frac{\delta^{2} \Psi}{\delta \hat{f}(2) \delta \hat{f}(1)}=D(1,2) \\
& =G^{-1}(1,3) G^{-1}(2,4) C(3,4)
\end{aligned}
$$

Here $G^{-1}(1,2)$ is the reverse Green's function defined by the relation

$$
G(1,3) G^{-1}(3,2)=\delta(1-2)
$$

The function $D(1,2)$ can be treated as a variance of effective random forces describing averaged effect on given mode from other modes due to nonlinear intermode interactions.

Relation (2.24) can be rewritten in the form of Wyld's equation obtained previously by summing up the perturbbation-theory series [8]

$$
C(1,2)=G(1,3) G(2,4) D(3,4)
$$


To find $G^{-1}$ and $D$ let us act on this equation by operators $\delta / \delta \hat{\eta}(4)$ and $\delta / i \delta \hat{f}(4)$.

In the limit $\eta \rightarrow 0$ and $f \rightarrow 0$ we will have

$$
\begin{aligned}
& \frac{\delta^{2} \Psi}{i \delta \hat{f}(1) \delta \hat{\eta}(4)}=G^{-1}(1,4)=L^{(0)}(1,4)-\Sigma(1,4) \\
& \Sigma(1,4)=\frac{1}{2} V(1 \mid 2,3) \frac{\delta}{\delta \hat{\eta}(4)} \cdot \frac{\delta^{2} \ln W}{\delta \eta(2) \delta \eta(3)} \\
& \frac{\delta^{2} \Psi}{\delta \hat{f}(4) \delta \hat{f}(1)}=D(1,4)=D^{(0)}(1,4)+D^{(1)}(1,4) \\
& D^{(1)}(1,4)=\frac{1}{2} V(1 \mid 2,3) \frac{\delta}{i \delta \hat{f}(4)} \cdot \frac{\delta^{2} \ln W}{\delta \eta(2) \delta \eta(3)}
\end{aligned}
$$

here $\Sigma(1,2)$ and $D^{(1)}(1,2)$ are corrections to the terms of molecular viscosity and covariance of external random forces induced by an influence of turbulent mixing.

To obtain the equation for velocity field variance, which describes the energy spectrum of turbulent pulsations, let us act to Equation (2.13) by operator $\delta / i \delta \eta(4)$ In the limit $\eta \rightarrow 0, f \rightarrow 0$ we get

$$
\begin{aligned}
& L^{(0)}(1,2) C(2,4)+\frac{1}{2} V(1 \mid 2,3) C(2,3,4) \\
& -D^{(0)}(1,2) G(2,4)=0
\end{aligned}
$$

here $C(2,3,4)$ is the three-point statistical moment of third order

$$
C(2,3,4)=-\frac{\delta}{i \delta \eta(4)} \cdot \frac{\delta^{2} \ln W}{\delta \eta(2) \delta \eta(3)}
$$

To calculate this function, one should apply Equations (2.18) and (2.19) and the identity

$$
\frac{\delta}{\delta \eta(1)}=\frac{\delta \hat{\eta}(2)}{\delta \eta(1)} \cdot \frac{\delta}{\delta \hat{\eta}(2)}+\frac{\delta \hat{f}(2)}{\delta \eta(1)} \cdot \frac{\delta}{\delta \hat{f}(2)}
$$

As the result, the nonlinear term in (2.29) appears to be presented in the following form

$$
\begin{aligned}
\frac{1}{2} V(1 \mid 2,3) C(2,3,4) & =-\Sigma(1,2) C(2,4) \\
& -G(1,2) D^{(1)}(2,4)
\end{aligned}
$$

The obtained representation of nonlinear term in the equation for velocity field variance is exact one, since, when finding Equation (2.32), no approximation has been made.

Equation (2.32) needs for some comments. Within the framework of phenomenological approach to closure problem, this term is treated as a turbulent viscosity and, with assumption of the gradient hypothesis type, it is commonly written as

$$
\nabla^{(1)}\left[v_{T}(1,2) \nabla^{(2)} C(2,4)\right]
$$

where the integral kernel $v_{T}(1,2)$ is the turbulent viscosity which shape can be found from the experimental data by the trial-and-error method. As the simplest example it may be the choice $v_{T}(1,2)=v_{T} \delta(1-2)$, where $v_{T}$ is some empirical constant. Another possibility of choice the representation for turbulent viscosity is $v_{T}(1,2)=v_{T}\left(\boldsymbol{r}_{1}-\boldsymbol{r}_{2}\right) \delta\left(t_{1}-t_{2}\right)$ known as "Marcovian approximation". The result (2.32) means that the interpretation of nonlinear term as turbulent viscosity is unsafficient, and, when applying the phenomenological closure scheme, one has to account for influence of effective random forces being subject to normal distribution with the covariance $D^{(1)}$.

\section{Equations for Functions $\Sigma$ and $D^{(1)}$ and the Closuring Procedure}

The set of Equations (2.27) and (2.28) is not close since it includes two unknown functions $\Sigma$ and $D^{(1)}$. To find these functions, one needs to calculate the functional derivatives of Wyld's Equation (2.26) with respect to $\hat{\eta}$ and $\hat{f}$. The functional derivatives of Green's function $G$ emerged can be found from the formula obtained after differentiating relation (2.18) with respect to $\hat{\eta}$ and $\hat{f}$. If the extremality condition $(\eta \rightarrow 0)$ is satisfied, one gets

$$
\begin{aligned}
\frac{\delta G(1,2)}{\delta \hat{\eta}(3)} & =-G(1,4) \frac{\delta^{3} \Psi}{i \delta \hat{f}(4) \delta \hat{\eta}(5) \delta \hat{\eta}(3)} G(5,2) \\
& =-G(1,4) \Gamma(4 \mid 3,5) G(5,2)
\end{aligned}
$$

In a similar manner

$$
\begin{aligned}
\frac{\delta G(1,2)}{i \delta \hat{f}(3)} & =-G(1,4) \frac{\delta^{3} \Psi}{i \delta \hat{f}(3) i \delta \hat{f}(4) \delta \hat{\eta}(5)} G(5,2) \\
& =-G(1,4) \Gamma(4 \mid 3,5) G(5,2) \\
& -C(1,4) \Gamma(3 \mid 4,5) G(5,2)
\end{aligned}
$$

Differentiating Wyld's Equation (2.26) with respect to $\hat{\eta}$ and $\hat{f}$ leads to following relations

$$
\begin{aligned}
\frac{\delta C(1,2)}{\delta \hat{\eta}(3)} & =-2 G(1,4) \Gamma(4 \mid 3,5) G(5,2) \\
& -G(1,4) G(2,5) \Gamma(4,5 \mid 3) \\
\frac{\delta C(1,2)}{i \delta \hat{f}(3)} & =-2 G(1,4) \Gamma(3,4 \mid 5) C(5,2) \\
& -G(1,4) G(2,5) \Gamma(3,4,5)
\end{aligned}
$$

where we introduced three new functions 


$$
\begin{aligned}
\Gamma(1 \mid 2,3) & =\frac{\delta^{3} \Psi}{i \delta \hat{f}(1) \delta \hat{\eta}(2) \delta \hat{\eta}(3)}=\frac{\delta G^{-1}(1,2)}{\delta \hat{\eta}(3)} \\
\Gamma(1,2 \mid 3) & =\frac{\delta^{3} \Psi}{i \delta \hat{f}(1) i \delta \hat{f}(2) \delta \hat{\eta}(3)} \\
& =-\frac{\delta D(1,2)}{\delta \hat{\eta}(3)}=\frac{\delta G^{-1}(1,3)}{i \delta \hat{f}(2)} \\
\Gamma(1,2,3) & =\frac{\delta^{3} \Psi}{i \delta \hat{f}(1) i \delta \hat{f}(2) i \delta \hat{f}(3)}=-\frac{\delta D(1,2)}{i \delta \hat{f}(3)}
\end{aligned}
$$

In the diagramm technique of quantum field theory these functions are referred to as vertices.

The resulting expressions for desired functions $\Sigma$ and $D^{(1)}$ take the form

$$
\begin{aligned}
\Sigma(1,2) & =-\frac{1}{2} V(1 \mid 3,4) G(3,5) \\
& \times[2 C(4,6) \Gamma(5 \mid 6,2)+G(4,6) \Gamma(5,6 \mid 2)]
\end{aligned}
$$

and

$$
\begin{aligned}
& D^{(1)}(1,2)=\frac{1}{2} V(1 \mid 3,4) \\
& \times[2 G(3,5) C(4,6) \Gamma(2,5 \mid 6) \\
& +G(3,5) G(4,6) \Gamma(2,5,6)]
\end{aligned}
$$

The set of equations obtained is exact one. However, this set is not closed because it contains the vertices of three types. In turn, using functional differentiating Equations (2.27) and (2.28) with respect to $\hat{\eta}$ and $\hat{f}$, one can obtain equations which will contain 4-th derivatives of characteristic functional $\Psi$. If to continue this procedure, it will arise an infinite chain of equations for vertices of various orders being similar to the chain for statistic moments by Keller and Freidman. To cut off this chain in the way that differs from commonly used closure schemes, we apply the low-order perturbation theory approximation for vertices by putting

$$
\Gamma(1 \mid 2,3)=V(1 \mid 2,3), \quad \Gamma(1,2 \mid 3)=\Gamma(1,2,3)=0
$$

Such approximation for $\Sigma$ and $D^{(1)}$ after substituteing those into the equations for $G^{-1}$ and $C$ and solving the set obtained will correspond to summing up a certain infinite subsequence of total perturbation-theory series for the quantities desired.

In the case $\hat{\eta}=0$ (averaged velocity vanishes when $f=0$ ), we come to the closed set of equations for functions $G$ and $C$

$$
\begin{aligned}
& G^{-1}(1,2)=L^{(0)}(1,2)-\Sigma(1,2) \\
& \Sigma(1,2)=12 V(1 \mid 3,4) G(3,5) C(4,6) V(5 \mid 6,2)
\end{aligned}
$$

and

$$
\begin{aligned}
& C(1,2)=G(1,3) G(2,4)\left[D^{(0)}(3,4)+D^{(1)}(3,4)\right] \\
& D^{(1)}(1,2)=\frac{1}{2} V(1 \mid 3,4) C(3,5) C(4,6) V(2 \mid 5,6)
\end{aligned}
$$

The set of tensor Equations (3.9)-(3.10) can be essentiely simplified by using the condition of velocity field solenoidality, $\partial_{\alpha} u_{\alpha}=0$, from which it follows that functions $G_{\alpha_{1} \alpha_{2}}(1,2)$ and $C_{\alpha_{1} \alpha_{2}}(1,2)$ have the form

$$
\begin{aligned}
& G_{\alpha_{1} \alpha_{2}}(1,2)=P_{\alpha_{1} \alpha_{2}}(1,3) G(3,2), \\
& C_{\alpha_{1} \alpha_{2}}(1,2)=P_{\alpha_{1} \alpha_{2}}(1,3) C(3,2)
\end{aligned}
$$

With account for the unimodality property of the transversal projection operator

$$
P_{\alpha_{1} \alpha_{3}}(1,3) P_{\alpha_{3} \alpha_{2}}(3,2)=P_{\alpha_{1} \alpha_{2}}(1,2)
$$

and from Wyld's Equation (2.26) it also follows

$$
\begin{aligned}
& G_{\alpha_{1} \alpha_{2}}^{-1}(1,2)=P_{\alpha_{1} \alpha_{2}}(1,3) G^{-1}(3,2), \\
& D_{\alpha_{1} \alpha_{2}}(1,2)=P_{\alpha_{1} \alpha_{2}}(1,3) D(3,2)
\end{aligned}
$$

Thus, the problem is formulated in terms of two scalar functions that depend on modulus of vector coordinates.

\section{Equations in the Space of Fourier Transforms}

After going to the space of Fourier-transforms, for the operator of transversal projection, vertex, Green's function, and velocity covariance one gets

$$
\begin{gathered}
P_{\alpha \beta}(\boldsymbol{k})=\delta_{\alpha \beta}-\frac{k_{\alpha} k_{\beta}}{k^{2}} \\
V_{\alpha \beta \gamma}\left(\boldsymbol{k}, \omega \mid \boldsymbol{q}, \omega^{\prime} ; \boldsymbol{k}-\boldsymbol{q}, \omega-\omega^{\prime}\right)=-i\left(\delta_{\alpha \beta} k_{\gamma}+\delta_{\alpha \gamma} k_{\beta}\right) \\
G_{\alpha \beta}(\boldsymbol{k}, \omega)=P_{\alpha \beta}(\boldsymbol{k}) G\left(k^{2}, \omega\right) \\
C_{\alpha \beta}(\boldsymbol{k}, \omega)=P_{\alpha \beta}(\boldsymbol{k}) C\left(k^{2}, \omega\right)
\end{gathered}
$$

(while performing the Fourier transformation for vertex (2.5) the operators of transveral projection $P_{\alpha \beta}(\boldsymbol{k})$ were changed by the Kronecker delta $\delta_{\alpha \beta}$ due to the fact that in all formulas one only meets the convolution

$$
P_{\alpha \beta}(\boldsymbol{k}) V_{\beta \gamma \delta}\left(\boldsymbol{k}, \omega \mid \boldsymbol{q}, \omega^{\prime} ; \boldsymbol{k}-\boldsymbol{q}, \omega-\omega^{\prime}\right)
$$

and there exists the identity

$$
P_{\alpha \beta}(\boldsymbol{k}) P_{\beta \gamma}(\boldsymbol{k})=P_{\alpha \gamma}(\boldsymbol{k}) \equiv P_{\alpha \beta}(\boldsymbol{k}) \delta_{\beta \gamma}
$$

- the property of unimodality.

Due to the isotropy of the system considered the tensor $\Sigma_{\alpha \beta}$ has the form

$$
\Sigma_{\alpha \beta}(\boldsymbol{q}, \omega)=\delta_{\alpha \beta} \Sigma\left(q^{2}, \omega\right)+q_{\alpha} q_{\beta} \Sigma^{(1)}\left(q^{2}, \omega\right)
$$

and, since the operator $\Sigma_{\alpha \beta}$ is convoluted with the op- 
erator of transversal projection, the term proportional to $\Sigma^{(1)}$ gives no contribution and can be omitted.

In the Fourier space Formula (3.9) for $\Sigma_{\alpha \beta}$ takes the form

$$
\begin{aligned}
\Sigma_{\alpha \beta}(\boldsymbol{k}, \omega)= & \int \frac{\mathrm{d} \boldsymbol{q}}{(2 \pi)^{d}} \frac{\mathrm{d} \omega^{\prime}}{2 \pi} A_{\alpha \beta}(\boldsymbol{k}, \boldsymbol{q}) \\
& \cdot G\left(q^{2}, \omega^{\prime}\right) C\left((\boldsymbol{k}-\boldsymbol{q})^{2}, \omega-\omega^{\prime}\right)
\end{aligned}
$$

where $d$ is the space dimension and

$$
A_{\alpha \beta}(\boldsymbol{k}, q)=V_{\alpha \gamma \delta}(\boldsymbol{k}) P_{\gamma \gamma^{\prime}}(\boldsymbol{q}) P_{\delta \delta^{\prime}}(\boldsymbol{k}-\boldsymbol{q}) V_{\gamma^{\prime} \delta^{\prime} \beta}(\boldsymbol{q})
$$

The relevant calculation gives

$$
A_{\alpha \beta}(\boldsymbol{k}, \boldsymbol{q})=-\frac{k^{2} q^{2}-(\boldsymbol{k} \cdot \boldsymbol{q})^{2}}{(\boldsymbol{k}-\boldsymbol{q})^{2}} \delta_{\alpha \beta}+\frac{2(\boldsymbol{k} \cdot \boldsymbol{q})}{q^{2}} q_{\alpha} q_{\beta}
$$

The correction to the variance of effective random force is calculated symilarly. According to Equation (3.10) the Fourier-transform of this quantity has the form

$$
\begin{aligned}
& D_{\alpha \beta}^{(1)}(\boldsymbol{k}, \omega)=-\int \frac{\mathrm{d} \boldsymbol{q}}{(2 \pi)^{d}} \frac{\mathrm{d} \omega^{\prime}}{2 \pi} B_{\alpha \beta}(\boldsymbol{k}, q) \\
& \cdot C\left(q^{2}, \omega^{\prime}\right) C\left((\boldsymbol{k}-\boldsymbol{q})^{2}, \omega-\omega^{\prime}\right)
\end{aligned}
$$

where

$$
\begin{aligned}
B_{\alpha \beta}(\boldsymbol{k}, \boldsymbol{q}) & =V_{\alpha \gamma \delta}(\boldsymbol{k}) P_{\gamma \gamma^{\prime}}(\boldsymbol{q}) P_{\delta \delta^{\prime}}(\boldsymbol{k}-\boldsymbol{q}) V_{\beta \gamma^{\prime} \delta^{\prime}}(-\boldsymbol{k}) \\
& =-2 A_{\alpha \beta}(\boldsymbol{k}, \boldsymbol{q})
\end{aligned}
$$

Thus, in combination with the equations

$$
\begin{aligned}
& G^{-1}\left(k^{2}, \omega\right)=-i \omega+v_{0} k^{2}-\Sigma\left(k^{2}, \omega\right), \\
& C\left(k^{2}, \omega\right)=G\left(k^{2}, \omega\right) D\left(k^{2}, \omega\right) G\left(k^{2},-\omega\right)
\end{aligned}
$$

one comes to a closed set of equations for two scalar functions $G$ and $C$ of two scalar variables $k^{2}$ and $\omega$.

\section{Conclusions}

One of the main exactly obtained results is a statement that a traditional treatment of nonlinear term in the equation for velocity covariance as turbulent viscosity is not safficient in modeling this term within the framework of phenomenological approach, and it should be accounted for additional term that describes the contribution from effective random forces. The proposed expression for this term may occur to be useful in finding the energy spectrum of turbulent pulsations

$$
E(k)=\frac{d-1}{2} \int \frac{\mathrm{d} \omega}{2 \pi} C\left(k^{2}, \omega\right)
$$

The equation of energy balance in the wave-number space can be obtained after Fourier transformation of the equation for velocity covariance and integrating over $\omega$. In this case the nonlinear term in the equation obtained may be written in the form of divergence (in the space of wave numbers) of a certain vector treated as the energy flux along the wave-number spectrum. From the dimensionality arguments and the assumption on locality of intermode interactions in the wave-number space it follows that the spectral energy flux defines the spectrum shape, however, unlike the Kolmogorov's theory, this quantity is not a constant due to dissipation effects.

The closed set of equations for two scalar functions $C$ and $G$ of two scalar variables obtained by applying the perturbation theory approximation for high-order statistical moments proves to be more simple in numerical sumulation the turbulence problem. When solving these equations, the renormalization-group method may appear to be useful since this method is based on the property of locality of intermode interactions in the wave-number space (see, [18] and, for example, [19]).

\section{REFERENCES}

[1] A. S. Monin and A. M. Yaglom, "Statistical Hydrodynamics. Theory of turbulence, V.1 (in Russian)," Hydrometeoizdat, St-Petersburg, 1992.

[2] M. I. Vishik and A. V. Fursikov, "Mathematical Problems in Statistical Hydrodynamics (in Russian)," Nauka, Moscow, 1980.

[3] L. V. Keller and A. A. Friedman, "Differentialgleichungen fur die Turbulente Bewegung einer kompressiblen Flussigkeit," Proceedings of the 1st International Congress for Applied Mechanics, Delft, 1924, pp. 395-405.

[4] N. N. Bogolyubov, "Problems of Dynamic Theory in Statistical Physics (in Russian)," GITTL, Kiev, 1946.

[5] Y. Ogura, "Energy Transport in a Normally Distributed and Isotropic Turbulent Velocity Field in Two Dimensions," Physics of Fluids, Vol. 5, No. 4, 1962, pp. 395 401. doi:10.1063/1.1706631

[6] R. H. Kraichnan, "The Structure of Isotropic Turbulence at Very High Reynolds Numbers," Journal of Fluid Mechanics, Vol. 5, No. 4, 1959, pp. 497-543. doi: $10.1017 / \mathrm{S} 0022112059000362$

[7] S. F. Edwards, "The Statistical Dynamics of Homogeneous Turbulence," Journal of Fluid Mechanics, Vol. 18, No. 2, 1964, pp. 239-293. doi:10.1017/S0022112064000180

[8] H. W. Wyld, "Formulation of the Theory of Turbulence in an Incompressible Fluid," Annals of Physics, Vol. 14, No. 2, 1961, pp. 143-165. doi:10.1016/0003-4916(61)90056-2

[9] E. V. Teodorovich, "The Use of the Renormalization Group Method to Describe Turbulence (a Review)," Izvestia, Amospheric and Oceanic Physics, Vol. 29, No. 2, 1993, pp. 149-163.

[10] L. T. Adzhemyan, N. V. Antonov and A. N. Vasil'ev, "Quantum-Field Renormalization Group in the Theory of 
Hydrodynamic Turbulence," Physics, Uspekhi, Vol. 39, No. 12, 1996, pp. 1193-1219.

doi:10.1070/PU1996v039n12ABEH000183

[11] A. N. Kolmogorov, "Local Structure of Turbulence in Incompressible Fluid at Very Large Reynolds Numbers (in Russian)," Soviet Physics, Doklady, Vol. 39, No. 4, 1941, pp. 299-303.

[12] B. B. Kadomtsev, "The Plasma Turbulence," Academic Press, London, New York, 1965.

[13] V. I. Belinicher and V. S. L'vov, "Scale-Invariant Theory of Developed Hydrodynamic Turbulence," Journal of Experimental and Theoretical Physics (JETP), Vol. 66, No. 2, 1987, pp. 303-313.

[14] E. V. Teodorovich, "Role of Local and Nonlocal Interactions in the Formation of the Developed Turbulence Regime," Fluid Dynamics, Vol. 25, No. 4, 1990, pp. 522528. doi:10.1007/BF01049856

[15] R. M. Lewis and R. H. Kraichnan, "A Space-Time Functional Formalism for Turbulence," Communication on
Pure and Applied Mathematics, Vol. 15, No. 2, 1962, pp. 397-411. doi:10.1002/cpa.3160150403

[16] E. V. Teodorovich, "Application of the Methods from Quantum-Field Theory," In the Monograph: A. S. Monin and A. M. Yaglom, Statistical Hydrodynamics. Theory of Turbulence. V.2, Sec. 29.4 (in Russian), St-Petersburg, Hydrometeoizdat, 1996.

[17] E. V. Teodorovoch, "Diagram Equations of the Theory of Fully Developed Turbulence," Theoretical and Mathematical Physics, Vol. 101, No. 1, 1994, pp. 1177-1183.

[18] E. V. Teodorovich, "The Renormalization-Group Method in Problems of Mechanics," Journal of Applied Mathematics and Mechanics (PMM), Vol. 68, No. 2, 2004, pp. 299-326. doi:10.1016/S0021-8928(04)90029-9

[19] E. V. Teodorovich, "Renormalization-Group Approach to Solving the Equation of Nonlinear Transfer," Journal of Physics A: Mathematical and Theoretical, Vol. 42, No. 15, 2009, Article ID: 155202. 